\title{
Object segmentation by Oriented Image Foresting Transform with connectivity constraints
}

\author{
Lucy A. C. Mansilla* and Paulo A. V. Miranda \\ Department of Computer Science, \\ Institute of Mathematics and Statistics - University of São Paulo (IME-USP), \\ 05508-090, São Paulo, SP, Brazil. \\ E-mail: \{lucyacm, pmiranda\}@ vision.ime.usp.br.
}

\begin{abstract}
Global properties, such as connectivity, shape constraints and boundary polarity, are useful high-level priors for image segmentation, allowing its customization for a given target object. In this work, we introduce a new method called Connected Oriented Image Foresting Transform (COIFT), which provides global optimum solutions according to a graph-cut measure, subject to the connectivity constraint in Oriented Image Foresting Transform (OIFT), ensuring the generation of connected objects, as well as allowing the simultaneous control of the boundary polarity. While the use of connectivity constraints in other frameworks, such as in the min-cut/max-flow algorithm, leads to an NP-Hard problem, COIFT conserves the low complexity of the OIFT algorithm. Experiments show that COIFT can considerably improve the segmentation of objects with thin and elongated parts, for the same number of seeds in segmentation based on markers.
\end{abstract}

\section{INTRODUCTION}

Object segmentation is one of the most fundamental and challenging problems in image processing and computer vision. In this work, we focus on seed-based methods for interactive image segmentation [2]-[6], where the user provides a partial labeling by drawing scribbles on the image (seed pixels). A graph derived from the image is then partitioned among the different labeled seeds according to some energy formulation, which can be roughly described in a unified manner according to a common framework, sometimes referred to as, Generalized Graph Cut (GGC) [7], [8]. Within this framework, there are two important classes of energy formulations, the $\varepsilon_{1}$ - and $\varepsilon_{\infty}$-minimization problems [7], the former including the min-cut/max-flow algorithm [3], whereas the latter class encompasses methods, such as watersheds [6], fuzzy connectedness [5], and some cases of image foresting transform (IFT) [9].

Connectedness is an important global topology property, which can be used as a high-level prior for object segmentation. For a given binary image that represents the segmentation result, in this work, we consider a connected component as a maximal set of pixels, such that there are paths composed by adjacent pixels (ex: 4-neighborhood or 8-neighborhood) interconnecting all its elements and passing exclusively within the object. In this context, the seed-based methods can be classified into three groups, according to their level of Connectedness: (1) In the first group, we have methods that do

\footnotetext{
* This work relates to the PhD thesis of the first author [1].
}

not guarantee any level of connectedness (Figures $1 \mathrm{a}-\mathrm{b}$ ). In the graph cut (GC) community, this is usually referred to as the disconnection problem of GC, when the source and sink nodes are connected to all image pixels [3]. (2) In the second group, we have methods that guarantee that object's pixels are connected to some internal seed. However, note that the object could be composed by several disconnected components, as long as we have some object's seeds in each component (Figure 1F). The majority of methods belong to this class, including fuzzy connectedness and watershed from markers [5], [6]. (3) In the third group, we have methods that guarantee that the segmented object forms a single connected component in the image domain [10]-[13]. This is especially important when the target is a single object (Figure 1 d d).

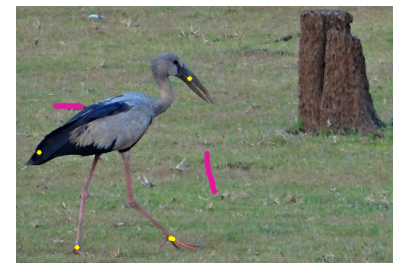

(a)

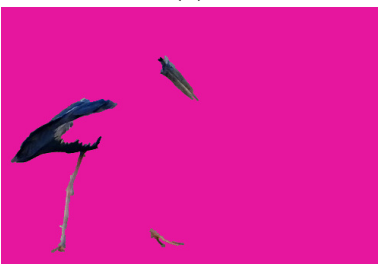

(c)

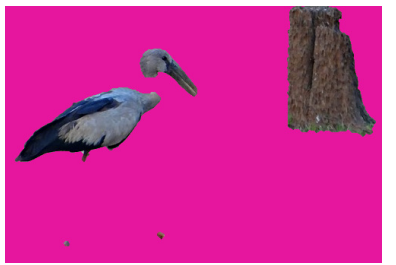

(b)

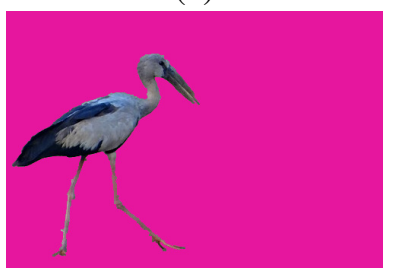

(d)
Fig. 1. (a) Input image with user selected seeds. (b) Segmentation by Graph cut showing the disconnection problem of an object region that is not marked by any seed on the right. (c) Segmentation by IFT resulting in disconnected components that are all marked by some object seed. (d) Segmentation by the proposed method producing a single connected component.

In this work, we use the term connectivity constraint to indicate methods from the third group. The $\varepsilon_{1}$-minimization among all objects satisfying the connectivity constraint was proved to be NP-Hard [10], [11]. Vicente et al. [10] propose a heuristic algorithm, named DijkstraGC, which merges the Dijkstra algorithm and graph cut. DijkstraGC is still slow, since it requires many calls to the maxflow algorithm. Other 
method, named Topology cuts, by Zeng et al. [11] also finds only an approximate solution to incorporate topology priors in the min-cut/max-flow algorithm. Nowozin and Lampert adopted a different approach solving a related optimization problem, which forces the output labeling to be connected in the framework of recent maximum a posteriori (MAP)-MRF linear program (LP) relaxations [12], [13].

In this $\mathrm{PhD}$ thesis, our main goal was to develop a new seedbased segmentation method that guarantees optimal results subject to the connectivity constraint in the $\varepsilon_{\infty}$-minimization problem of the GGC framework.

In Section III we review IFT and OIFT. In Sections III and IV we present and evaluate our proposed method, and our conclusions are stated in Section $\mathrm{V}$

\section{IMAGE GRAPH CONCEPTS}

A 2D/3D image can be interpreted as a weighted digraph $G=\langle\mathcal{V}, \mathcal{A}, \omega\rangle$, whose nodes $\mathcal{V}$ are the image pixels/voxels in its image domain $\mathcal{I} \subset \mathbb{Z}^{n}$, and whose arcs are the ordered pixel pairs $\langle s, t\rangle \in \mathcal{A}$. The digraph $G$ is symmetric if for any of its $\operatorname{arcs}\langle s, t\rangle \in \mathcal{A}$, the pair $\langle t, s\rangle$ is also an arc of $G$. Each arc $\langle s, t\rangle \in \mathcal{A}$ has a weight $\omega(\langle s, t\rangle)$, such as a dissimilarity measure between pixels $s$ and $t$ (e.g., $\omega(\langle s, t\rangle)=|I(t)-I(s)|$ for a single channel image with values given by $I(t))$. For a given image graph $G=\langle\mathcal{V}, \mathcal{A}, \omega\rangle$, a path $\pi=\left\langle t_{1}, t_{2}, \ldots, t_{n}\right\rangle$ is a sequence of adjacent pixels (i.e., $\left\langle t_{i}, t_{i+1}\right\rangle \in \mathcal{A}, i=1,2, \ldots, n-1$ ) with no repeated vertices $\left(t_{i} \neq t_{j}\right.$ for $\left.i \neq j\right)$. Other greek letters, such as $\tau$, can also be used to denote different paths. A path $\pi_{t}=\left\langle t_{1}, t_{2}, \ldots, t_{n}=t\right\rangle$ is a path with terminus at a pixel $t$. When we want to explicitly indicate the origin of the path, the notation $\pi_{s \sim t}=\left\langle t_{1}=s, t_{2}, \ldots, t_{n}=t\right\rangle$ may also be used, where $s$ stands for the origin and $t$ for the destination node. More generally, we can use $\pi_{\mathcal{S} \sim t}=\left\langle t_{1}, t_{2}, \ldots, t_{n}=t\right\rangle$ to indicate a path with origin restricted to a set $\mathcal{S}$ (i.e., $t_{1} \in \mathcal{S}$ ). A path is trivial when $\pi_{t}=\langle t\rangle$. A path $\pi_{t}=\pi_{s} \cdot\langle s, t\rangle$ indicates the extension of a path $\pi_{s}$ by an arc $\langle s, t\rangle$.

A predecessor map is a function $P$ that assigns to each pixel $t$ in $\mathcal{V}$ either some other adjacent pixel in $\mathcal{V}$, or a distinctive marker nil not in $\mathcal{V}-$ in which case $t$ is said to be a root of the map. A spanning forest is a predecessor map which contains no cycles - i.e., one which takes every pixel to nil in a finite number of iterations. For any pixel $t \in \mathcal{V}$, a spanning forest $P$ defines a path $\pi_{t}^{P}$ recursively as $\langle t\rangle$ if $P(t)=n i l$, and $\pi_{s}^{P} \cdot\langle s, t\rangle$ if $P(t)=s \neq n i l$.

\section{A. Image Foresting Transform (IFT)}

A connectivity function computes a value $f\left(\pi_{t}\right)$ for any path $\pi_{t}$, usually based on arc weights. A path $\pi_{t}$ is optimum if $f\left(\pi_{t}\right) \leq f\left(\tau_{t}\right)$ for any other path $\tau_{t}$ in $G$. By taking to each pixel $t \in \mathcal{V}$ one optimum path with terminus at $t$, we obtain the optimum-path value $V_{o p t}^{f}(t)$, which is uniquely defined by $V_{\text {opt }}^{f}(t)=\min _{\forall \pi_{t} \text { in } G}\left\{f\left(\pi_{t}\right)\right\}$.

The image foresting transform (IFT) [9] takes an image graph $G=\langle\mathcal{V}, \mathcal{A}, \omega\rangle$, and a path-cost function $f$; and assigns one optimum path to every pixel $t \in \mathcal{V}$ such that an optimumpath forest $P$ is obtained - i.e., a spanning forest where all paths $\pi_{t}^{P}$ for $t \in \mathcal{V}$ are optimal. However, $f$ must meet certain admission criteria, otherwise, the paths may not be optimal, as demonstrated in [14].

The cost of a trivial path $\pi_{t}=\langle t\rangle$ is usually based on a seed set $\mathcal{S}$, and the cost for non-trivial paths follow a path-extension rule. The path-cost function $f_{\max }^{\| \mathcal{S}}$ is a Monotonic-Incremental cost function (MI) and, consequently, is guaranteed to provide optimum results in the IFT framework [9]. This function will be important in COIFT. Note that $f_{\max }^{\| \mathcal{S}}$ processes anti-parallel $\operatorname{arcs}\langle t, s\rangle$ along the path, which requires a symmetric digraph.

$$
\begin{aligned}
f_{\max }^{\| \mathcal{S}}(\langle t\rangle) & = \begin{cases}-1 & \text { if } t \in \mathcal{S} \\
+\infty & \text { otherwise }\end{cases} \\
f_{\max }^{\| \mathcal{S}}\left(\pi_{s} \cdot\langle s, t\rangle\right) & =\max \left\{f_{\max }^{\| \mathcal{S}}\left(\pi_{s}\right), \omega(\langle t, s\rangle)\right\}
\end{aligned}
$$

The IFT algorithm computes a path-cost map $V$, which converges to $V_{o p t}^{f}$ if $f$ is a MI function [9], [14]. It is also optimized in handling infinite costs, by storing in its priority queue $\mathcal{Q}$ only the nodes with finite-cost path, assuming that $V_{o p t}^{f}(t)<+\infty$ for all $t \in \mathcal{V}$.

\section{B. Oriented Image Foresting Transform (OIFT)}

Let $G$ be a strongly connected and symmetric digraph, where the weights $\omega(\langle s, t\rangle)$ are a combination of an undirected dissimilarity measure $\delta(\langle s, t\rangle)$ between neighboring pixels $s$ and $t$, multiplied by an orientation factor, i.e., $\omega(\langle s, t\rangle)=$ $\delta(\langle s, t\rangle) \times(1+\alpha)$ if $I(s)>I(t), \omega(\langle s, t\rangle)=\delta(\langle s, t\rangle) \times(1-\alpha)$ if $I(s)<I(t)$ and $\omega(\langle s, t\rangle)=\delta(\langle s, t\rangle)$ otherwise, where $\alpha \in[-1,1]$. Different procedures can be adopted for $\delta(\langle s, t\rangle)$, as discussed in [15], [16], such as the absolute value of the difference of image intensities (i.e., $\delta(\langle s, t\rangle)=|I(s)-I(t)|)$. Note that we usually have $\omega(\langle s, t\rangle) \neq \omega(\langle t, s\rangle)$ when $\alpha \neq 0$. For colored images, a reference map should be considered for $I(t)$ or $\alpha$ must be set to zero [17].

OIFT is build upon the IFT framework by considering the following path function in a symmetric digraph:

$$
\begin{aligned}
f^{\sigma^{\top}}(\langle t\rangle) & = \begin{cases}-1 & \text { if } t \in \mathcal{S}_{1} \cup \mathcal{S}_{0} \\
+\infty & \text { otherwise }\end{cases} \\
f^{\sigma^{\top}}\left(\pi_{r \sim s} \cdot\langle s, t\rangle\right) & = \begin{cases}\omega(\langle s, t\rangle) & \text { if } r \in \mathcal{S}_{1} \\
\omega(\langle t, s\rangle) & \text { otherwise }\end{cases}
\end{aligned}
$$

where $\mathcal{S}_{1}$ and $\mathcal{S}_{0}$ denote, respectively, the set of seeds selected inside and outside the object to be segmented.

The segmented object $\mathcal{O}^{P}$ by OIFT is defined from the forest $P$ computed by the IFT algorithm, with $f^{\sigma^{7}}$, by taking as object pixels the set of pixels that were conquered by paths rooted in $\mathcal{S}_{1}$, i.e., $\mathcal{O}^{P}=\left\{t \in \mathcal{V} \mid \pi_{t}^{P}=\tau_{\mathcal{S}_{1} \sim t}\right\}$. For $\alpha>0$, the segmentation by OIFT favors transitions from bright to dark pixels, and $\alpha<0$ favors the opposite orientation.

The optimality of $\mathcal{O}^{P}$ by OIFT is supported by the maximization of an energy criterion of cut in graphs involving arcs from object to background pixels $\mathcal{C}\left(\mathcal{O}^{P}\right)$ (outer-cut boundary) [17], [18].

$$
\begin{aligned}
& \mathcal{C}(\mathcal{O})=\{\langle s, t\rangle \in \mathcal{A} \mid s \in \mathcal{O} \text { and } t \notin \mathcal{O}\} \\
& E(\mathcal{O})=\min _{\langle s, t\rangle \in \mathcal{C}(\mathcal{O})} \omega(\langle s, t\rangle)
\end{aligned}
$$




\section{OIFT WITH CONNECTIVITY CONSTRAINTS}

\section{A. Connectivity Constraints}

An object $\mathcal{O}$ is connected if for any pair of vertices $p, q \in \mathcal{O}$ there is a path $\pi_{p \sim q}=\left\{t_{1}=p, t_{2}, \ldots, t_{i}, \ldots, t_{n}=q\right\}$, such that $t_{i} \in \mathcal{O}, 1 \leqslant i \leqslant n$. In this work, we introduced the concept of $\gamma$-connectivity in relation to a given set $\mathcal{S}_{1}$ of internal seeds, which is described by Definition 11, where $\mathcal{V}_{\odot}(c, \gamma)$ is a disk with center $c$ and radius $\gamma$, defined by $\mathcal{V}_{\odot}(c, \gamma)=\{t \in \mathcal{I} \mid\|t-c\| \leqslant \gamma\}$, such that $\|t-c\|$ is the Euclidean distance between vertices $t$ and $c$.

Definition 1 ( $\gamma$-connected object). For a given object $\mathcal{O}$ and set of seeds in its interior $\mathcal{S}_{1}$, let $R^{\gamma}(\mathcal{O})$ be the set of all vertices $t \in \mathcal{O}$, such that $t$ is the center of a disk with radius $\gamma$, which is completely contained in the object $\mathcal{O}$. That is, $R^{\gamma}(\mathcal{O})=\left\{t \in \mathcal{O} \mid \mathcal{V}_{\odot}(t, \gamma) \subseteq \mathcal{O}\right\}$. An object $\mathcal{O}$ is $\gamma$-connected in relation to $\mathcal{S}_{1}$ if the seeds in $\mathcal{S}_{1}$ are all in the same connected component of $R^{\gamma}(\mathcal{O})$. That is, for any pair of vertices $p, q \epsilon$ $\mathcal{S}_{1}$, there is a path $\pi_{p \sim q}=\left\{t_{1}=p, t_{2}, \ldots, t_{i}, \ldots, t_{n}=q\right\}$, such that $t_{i} \in R^{\gamma}(\mathcal{O}), 1 \leqslant i \leqslant n$ (Figure 2 ).

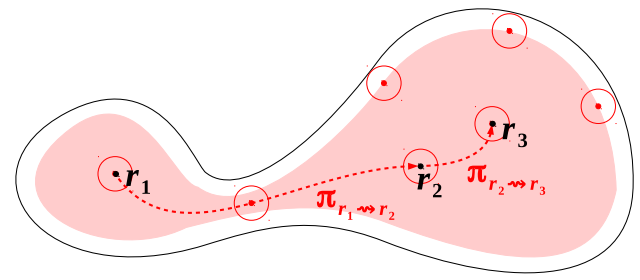

(a) A 1-connected object in relation to $\left\{r_{1}, r_{2}, r_{3}\right\}$

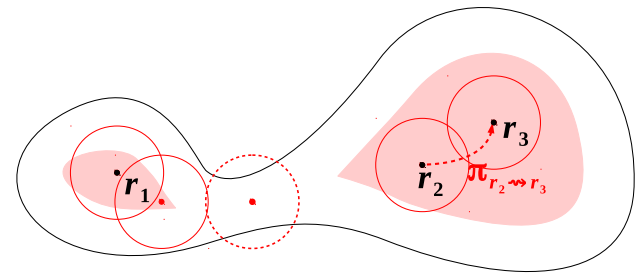

(b) A non-3-connected object in relation to $\left\{r_{1}, r_{2}, r_{3}\right\}$

Fig. 2. Examples of $\gamma$-connected and non- $\gamma$-connected objects. For an object $\mathcal{O}$ with $\mathcal{S}_{1}=\left\{r_{1}, r_{2}, r_{3}\right\}$, we have in: (a) A region $R^{\gamma}(\mathcal{O})$ with $\gamma=1$ (shaded region) such that $r_{1}, r_{2}$ and $r_{3}$ belong to the same connected component of $R^{\gamma}(\mathcal{O})$, identifying a 1-connected object. (b) A region $R^{\gamma}(\mathcal{O})$ with $\gamma=3$ (shaded region) such that $r_{1}$ does not belong to the same connected component of $r_{2}$ and $r_{3}$ in $R^{\gamma}(\mathcal{O})$, thus, not satisfying the constraint of 3-connectivity. Note that for $\gamma=1$, the paths $\pi_{r_{1} \sim r_{2}}, \pi_{r_{2} \sim r_{3}}$ and $\pi_{r_{1} \leadsto r_{3}}=\pi_{r_{1} \leadsto r_{2}} \cdot \pi_{r_{2} \leadsto r_{3}}$ are completely contained in $R^{\gamma}(\mathcal{O})$ and for $\gamma=3$ there are no paths that interconnect $r_{1}$ with $r_{2}$ (neither $r_{1}$ with $r_{3}$ ) and that are completely contained in $R^{\gamma}(\mathcal{O})$.

\section{B. Segmentation of a connected object via COIFT}

In order to resolve the disconnection problem in image segmentation, in [19] we successfully incorporated connectivity constraints in the OIFT approach, resulting in a novel method named Connected Oriented Image Foresting Transform (COIFT), which simultaneously handle boundary polarity and connectivity constraints.

Let $A$ be a given set of pixels, the optimum energy value using the set $A$ as internal seeds is denoted by
$E_{A}=\max _{\mathcal{O} \in \mathcal{U}\left(A, \mathcal{S}_{0}\right)} E(\mathcal{O})$. According to the same notation, $E_{\{t\}}$ denotes the optimum energy from a single internal seed, when $\mathcal{S}_{1}=\{t\}$. COIFT is supported by the following propositions:

Proposition 1. Let $E_{A \cup B}$ be the energy of a seed set $A \cup B$. The optimum energy $E_{A \cup B}$ among all objects in $\mathcal{U}\left(A \cup B, \mathcal{S}_{0}\right)$, satisfies $E_{A \cup B}=\min \left\{E_{A}, E_{B}\right\}$.

Proposition 2. For a given strongly connected and symmetric digraph $G$, and sets of seeds $\mathcal{S}_{1}$ and $\mathcal{S}_{0}$, such that $\mathcal{S}_{1}=\{t\}$ we have that $E_{\{t\}}=V_{o p t}^{f_{\max }^{\ell} \mathcal{S}_{0}}(t)$.

$V_{o p t}^{f_{\max }^{\| \mathcal{S}_{0}}}(t)$ is the cost of an optimum path by function $f_{\max }^{\ell} \mathcal{S}_{0}$ (Eq. 1). Hence, the first step of COIFT can be accomplished by computing $V_{\text {opt }}^{f_{\max }^{\| \mathcal{S}_{0}}}(t)=E_{\{t\}}$ for all $t \in \mathcal{V}$, which requires one execution of the IFT algorithm with $f_{\max }^{\| \mathcal{S}_{0}}$, using only the external seeds in $\mathcal{S}_{0}$ to initialize the costs of trivial paths. So, in a second step, we interconnect disconnected seeds in $\mathcal{S}_{1}$ through pixels with higher energy values of $E_{\{t\}}$ obtaining a new connected set of internal seeds $\mathcal{S}_{1}^{c}$. For that, we consider a particular case of the cost function employed by the Riverbed method [20] (see Figure 3.). In the last step, we simply

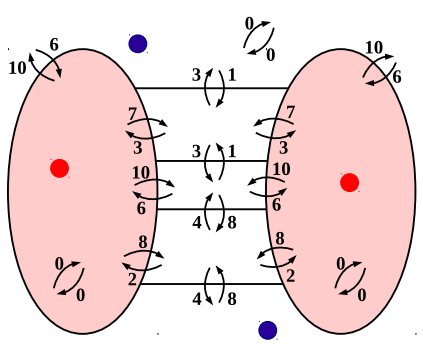

(a) $E(\mathcal{O})=7$

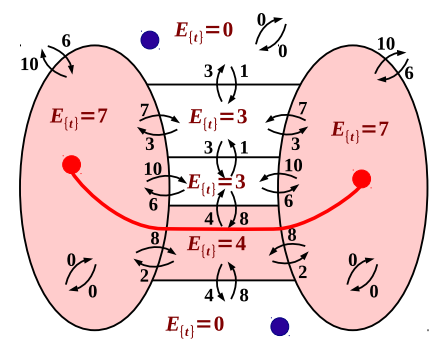

(b) $E(\mathcal{O})=4$
Fig. 3. Graph representation with segmentation results by: (a) OIFT. (b) COIFT. The riverbed path (red line) interconnects the disconnected internal seeds passing through regions with higher values of $E_{\{t\}}$.

compute the OIFT method with $f^{\sigma^{r}}$ from seed sets $\mathcal{S}_{1}^{c}$ and $\mathcal{S}_{0}$, generating a final result, which is guaranteed to be a connected object (Theorem 1 ).

Theorem 1 (Cut optimality by COIFT). For two given sets of seeds $\mathcal{S}_{1}$ and $\mathcal{S}_{0}$, let $\mathcal{U}\left(\mathcal{S}_{1}, \mathcal{S}_{0}\right)$ be the universe of all possible objects satisfying the seed constraints and let $\mathcal{U}_{c}\left(\mathcal{S}_{1}, \mathcal{S}_{0}\right)=\left\{\mathcal{O} \in \mathcal{U}\left(\mathcal{S}_{1}, \mathcal{S}_{0}\right) \mid G[\mathcal{O}]\right.$ is strongly connected $\}$, where $G[\mathcal{O}]$ is the subgraph of $G$ induced by $\mathcal{O}$, denote the universe of all possible connected objects satisfying the seed constraints. Any segmented object $\mathcal{O}_{c}$ computed by the COIFT algorithm (Algorithm 1) maximizes $E(\mathcal{O})$ (Eq. 4) among all possible segmentation results in $\mathcal{U}_{c}\left(\mathcal{S}_{1}, \mathcal{S}_{0}\right)$. That is, $E\left(\mathcal{O}_{c}\right)=\max _{\mathcal{O} \in \mathcal{U}_{c}\left(\mathcal{S}_{1}, \mathcal{S}_{0}\right)} E(\mathcal{O})$.

\section{Algorithm 1. - COIFT AlgORITHM}


INPUT:

Digraph $G=\langle\mathcal{I}, \mathcal{A}, \omega\rangle$, sets $\mathcal{S}_{1}$ and $\mathcal{S}_{0}$ of seeds and cost function $f^{\sigma^{\top}}$.

OUtPut: $\quad$ A component $\langle L, P, V\rangle$ containing label map $L$, spanning forest $P$ and path-cost map $V$.

AUXILIARY: Optimum-path cost map $V_{o p t}^{f_{\max }^{\# S_{0}}}$, energy map $\mathcal{E}: \mathcal{I} \rightarrow \mathbb{R}$ and set $\mathcal{S}_{1}^{c}$ of seeds.

1. Compute $\left\langle-,-, V_{\text {opt }}^{f_{\max }^{\# S_{0}}}\right\rangle \leftarrow \operatorname{IFT}\left(G, \varnothing, \mathcal{S}_{\mathbf{0}}, f_{\max }^{\# S_{0}}\right)$

2. For each $t \in \mathcal{I}$, do

3. $L \mathcal{E}(t) \leftarrow V_{\text {opt }}^{f_{\max }^{\# \mathcal{S}_{0}}}(t)$

4. Compute $\mathcal{S}_{1}^{c} \leftarrow N e w O b j \operatorname{Seeds}\left(G, \mathcal{S}_{1}, \mathcal{E}\right)$

5. Compute $\langle L, P, V\rangle \leftarrow \operatorname{IFT}\left(G, \mathcal{S}_{1}^{c}, \mathcal{S}_{\mathbf{0}}, f^{\sigma^{7}}\right)$

Return $\langle L, P, V\rangle$

\section{Algorithm 2. - NewObjSeeds Algorithm}

InPUT: $\quad$ Digraph $G=\langle\mathcal{I}, \mathcal{A}, \omega\rangle$, set $\mathcal{S}_{1}$ of seeds and

OUTPUT: energy map $\mathcal{E}: \mathcal{I} \rightarrow \mathbb{R}$.

AUXILIARY: Path forest $P_{\text {river }}$, cost function $f_{\text {river }}^{\prime}$ and variables $s^{*}, K_{1}$ and pred.

1. $s^{*} \leftarrow \arg \min _{t \in \mathcal{S}_{1}} \mathcal{E}(t)$

2. $K_{1} \leftarrow \max _{t \in \mathcal{I}} \mathcal{E}(t)$

3. For each $t \in \mathcal{I}$, do

4. $\quad\left\llcorner\quad \omega_{v}(t) \leftarrow K_{1}-\mathcal{E}(t)\right.$.

5. Compute $\left\langle-, P_{\text {river }},-\right\rangle \leftarrow \operatorname{IFT}\left(G,\left\{s^{*}\right\}, \varnothing, f_{\text {river }}^{\prime}\right)$

6. $\mathcal{S}_{1}^{c} \leftarrow \varnothing$

7. For each $t \in \mathcal{S}_{1}$, do

8. $\mid$ pred $\leftarrow t$

9. While pred $\neq$ nil, do

10. $\quad \mathcal{S}_{1}^{c} \leftarrow \mathcal{S}_{1}^{c} \cup\{$ pred $\}$

11. $\left[\right.$ pred $\leftarrow P_{\text {river }}($ pred $)$

Return $\mathcal{S}_{1}^{c}$

\section{Segmentation of a $\gamma$-connected object via COIFT}

In [21] we proposed a COIFT extension in order to segment $\gamma$-connected objects in relation to $\mathcal{S}_{1}$, for that we introduce a new parameter that controls the width of the connectivity regions, making COIFT more adaptable to different objects. In this configuration COIFT is called COIFT with adjustable width.

Let $E_{\mathcal{V}_{\odot}(t, \gamma)}$ be the optimum energy from a single disk $\mathcal{V}_{\odot}(t, \gamma)$, using $\mathcal{V}_{\odot}(t, \gamma)$ as internal seeds. From Propositions 11 and 2 we have $E_{\mathcal{V}_{\odot}(t, \gamma)}=\min _{p \in \mathcal{V}_{\odot}(t, \gamma)} E_{\{p\}}$ and $E_{\mathcal{V}_{\odot}(t, \gamma)}=\min _{p \in \mathcal{V}_{\odot}(t, \gamma)} V_{o p t}^{f_{\max }^{\# \mathcal{S}_{0}}}(p)$.

The algorithm of COIFT with adjustable width comprises the following three steps: (1) First, we compute $E_{\mathcal{V}_{\odot}(t, \gamma)}$ for all $t \in \mathcal{I}$ (Figures 4k,d). (2) Secondly, we interconnect disconnected seeds in $\mathcal{S}_{1}$, by computing paths passing through pixels $t$ with maximum energy $E_{\mathcal{V}_{\odot}(t, \gamma)}$, resulting in a new connected set of internal seeds $\mathcal{S}_{1}^{c}$ (Figure $4 \mathrm{k}$ ). For that, we use another particular case of the cost function employed by the Riverbed method [20]. In order to guarantee the width of the $\gamma$-connected object in relation to $\mathcal{S}_{1}$, we consider the disks centered at the set $\mathcal{S}_{1}^{c}$ to obtain a new connected set of internal seeds $\mathcal{S}_{1}^{\gamma c}$. (3) In the last step, we compute the OIFT method with $f^{\sigma^{\gamma}}$ from seed sets $\mathcal{S}_{1}^{\gamma c}$ and $\mathcal{S}_{0}$, generating a final result, which is guaranteed to be a $\gamma$-connected object (Theorem 2 .

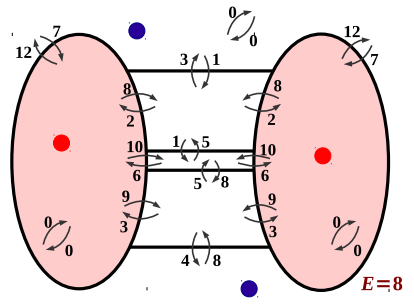

(a)

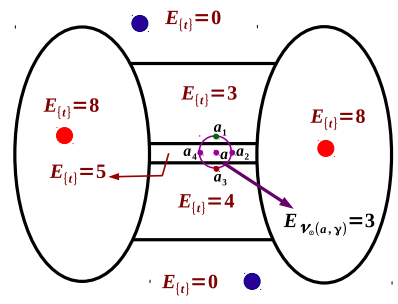

(c)

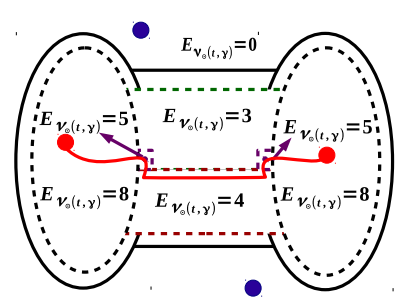

(e)

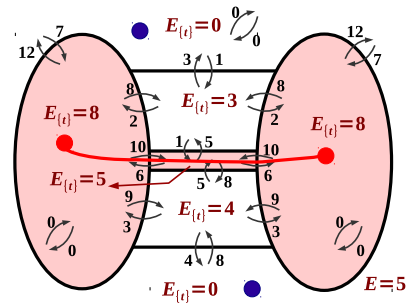

(b)

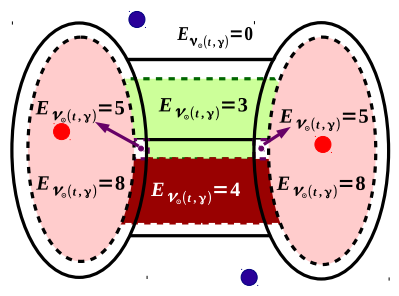

(d)

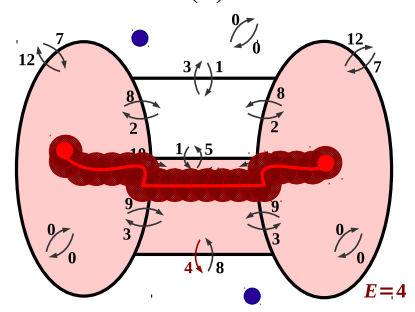

(f)
Fig. 4. Graph representation with segmentation results by: (a) OIFT. (b) COIFT $(\gamma=0)$, the riverbed path interconnecting the seeds and the values of $E_{\{t\}}$ for each region are shown in red. (c-f) COIFT with $\gamma=1.0$. In (c), we can observe how the energy of the disk $\mathcal{V}_{\odot}(a, \gamma)$ is computed for the pixel a, i.e., $E_{\mathcal{V}_{\odot}(a, \gamma)}=\min \left\{E_{\left\{a_{1}\right\}}, E_{\left\{a_{2}\right\}}, E_{\left\{a_{3}\right\}}, E_{\left\{a_{4}\right\}}, E_{\{a\}}\right\}=$ $\min \{3,5,4,5,5\}=3$. The colored regions in (d) represent the final energy regions $E_{\mathcal{V}}(t, \gamma)$ for all $t \in \mathcal{V}$. (e) The riverbed path interconnecting the internal seeds is shown. (f) A 1-connected object in relation to the initial set of internal seeds is obtained.

Theorem 2 (Cut optimality by COIFT with adjustable width). For two given set of seeds $\mathcal{S}_{1}$ and $\mathcal{S}_{0}$, let $\mathcal{U}_{c}^{\gamma}\left(\mathcal{S}_{1}, \mathcal{S}_{0}\right)=$ $\left\{\mathcal{O} \in \mathcal{U}\left(\mathcal{S}_{1}, \mathcal{S}_{0}\right) \mid \mathcal{O}\right.$ is $\gamma$-connected in relation to $\left.\mathcal{S}_{1}\right\}$ be the set of all $\gamma$-connected objects in relation to $\mathcal{S}_{1}$ satisfying the seed constraints (i.e., $\mathcal{U}_{c}^{\gamma}\left(\mathcal{S}_{1}, \mathcal{S}_{0}\right) \subseteq \mathcal{U}\left(\mathcal{S}_{1}, \mathcal{S}_{0}\right)$ ). Any segmented object $\mathcal{O}_{c}^{\gamma}$ computed by the COIFT-with-adjustablewidth algorithm (Algorithm 3 ) maximizes $E(\mathcal{O})(E q .4)$ among all possible segmentation results in $\mathcal{U}_{c}^{\gamma}\left(\mathcal{S}_{1}, \mathcal{S}_{0}\right)$. That is, $E\left(\mathcal{O}_{c}^{\gamma}\right)=\max _{\mathcal{O} \in \mathcal{U}_{c}^{\gamma}\left(\mathcal{S}_{1}, \mathcal{S}_{0}\right)} E(\mathcal{O})$.

Algorithm 3. - COIFT-WITH-ADJUSTABLE-WIDTH ALGORITHM

INPUT:

Digraph $G=\langle\mathcal{I}, \mathcal{A}, \omega\rangle$, sets $\mathcal{S}_{1}$ and $\mathcal{S}_{0}$ of seeds and cost function $f^{\sigma^{\prime}}$.

OutPuT: $\quad$ A component $\langle L, P, V\rangle$ containing label map $L$, spanning forest $P$ and path-cost map $V$.

AUXILIARY: Optimum-path cost map $V_{\text {opt }}^{f_{\max }^{\# S_{0}}}$, energy map $\mathcal{E}_{\odot}: \mathcal{I} \rightarrow \mathbb{R}$ and set $\mathcal{S}_{1}^{\gamma c}$ of seeds.

1. Compute $\left\langle-,-, V_{\text {opt }}^{f_{\max }^{\# S_{0}}}\right\rangle \leftarrow \operatorname{IFT}\left(G, \varnothing, \mathcal{S}_{\mathbf{0}}, f_{\max }^{\# S_{0}}\right)$

2. For each $t \in \mathcal{I}$, do 
3. $\quad L \quad \mathcal{E}_{\odot}(t) \leftarrow \min _{p \in \mathcal{V}_{\odot}(t, \gamma)} V_{o p t}^{f_{\max }^{\# \mathcal{S}_{0}}}(p)$

4. Compute $\mathcal{S}_{1}^{\gamma c} \leftarrow N e w D i s k O b j \operatorname{Seeds}\left(G, \mathcal{S}_{\mathbf{1}}, \mathcal{E}_{\odot}\right)$

5. Compute $\langle L, P, V\rangle \leftarrow \operatorname{IFT}\left(G, \mathcal{S}_{1}^{\gamma c}, \mathcal{S}_{0}, f^{\sigma^{\top}}\right)$

Return $\langle L, P, V\rangle$

\section{Algorithm 4. - NewDiskObjSeeds Algorithm}

InPUT: $\quad$ Digraph $G=\langle\mathcal{I}, \mathcal{A}, \omega\rangle$, set $\mathcal{S}_{1}$ of seeds and $\begin{array}{ll} & \text { energy map } \mathcal{E}_{\odot}: \mathcal{I} \\ \text { OUTPUT: } & \text { Set } \mathcal{S}_{1}^{\gamma c} \text { of seeds. }\end{array}$

AUXILIARY: Path forest $P_{\text {river }}$, cost function $f_{\text {river }}^{\prime}$, set $\mathcal{S}_{1}^{c}$ of seeds and variables $s^{*}, K_{2}$ and pred.

1. $s^{*} \leftarrow \arg \min _{t \in \mathcal{S}_{1}} \mathcal{E}_{\odot}(t)$

2. $K_{2} \leftarrow \max _{t \in \mathcal{I}} \mathcal{E}_{\odot}(t)$

3. For each $t \in \mathcal{I}$, do

4. $\quad\left\llcorner\quad \omega_{v}(t) \leftarrow K_{2}-\mathcal{E}_{\odot}(t)\right.$.

5. Compute $\left\langle-, P_{\text {river }},-\right\rangle \leftarrow \operatorname{IFT}\left(G,\left\{s^{*}\right\}, \varnothing, f_{\text {river }}^{\prime}\right)$

6. $\mathcal{S}_{1}^{c} \leftarrow \varnothing$

7. For each $t \in \mathcal{S}_{1}$, do

8. $\mid$ pred $\leftarrow t$

9. While pred $\neq$ nil, do

10. $\quad \mathcal{S}_{1}^{c} \leftarrow \mathcal{S}_{1}^{c} \cup\{$ pred $\}$

11. $\left[\right.$ pred $\leftarrow P_{\text {river }}($ pred $)$

12. $\mathcal{S}_{1}^{\gamma c} \leftarrow \varnothing$

13. For each $t \in \mathcal{S}_{1}^{c}$, do

14. $L \mathcal{S}_{1}^{\gamma c} \leftarrow \mathcal{S}_{1}^{\gamma c} \cup \mathcal{V}_{\odot}(t, \gamma)$

Return $\mathcal{S}_{1}^{\gamma c}$

In the PhD thesis, we also extended the theoretical analysis of our works [19] and [21] in order to improve the handling of ambiguities in the energy formulation of COIFT without affecting its theoretical results but improving the quality of the resulting segmentation.

Figure 5 shows an example of the segmentation by OIFT and COIFT for the same user-selected markers, making clear the advantages of COIFT.

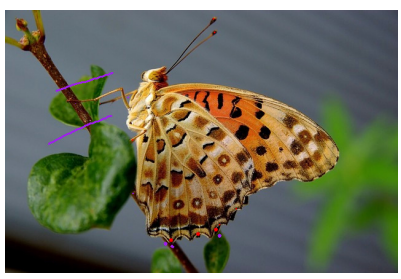

(a)

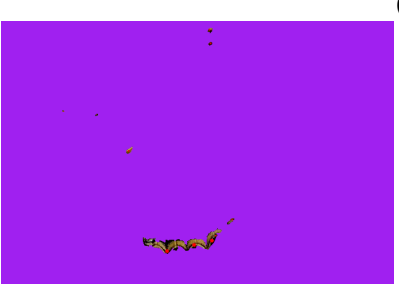

(b) OIFT

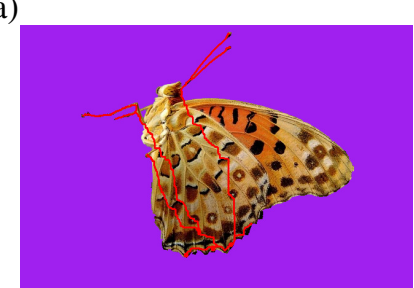

(c) COIFT with $\gamma=1.0$
Fig. 5. (a) Input image with user-selected markers. (b) A poor segmentation result is obtained by OIFT. (c) An improved segmentation result by COIFT.

\section{EXPERIMENTS}

In our experiments we compared COIFT against other methods with competitive running times (IRFC [5], OIFT [18],
ORFC+GC [22], [23]), to show how $\varepsilon_{\infty}$-minimization (or equivalently $E(\mathcal{O})$-maximization) based methods can benefit from the use of connectivity constraints. In order to stress the methods, we only considered the image-based weight assignment from [16] for $\delta(s, t)$, without any prior intensity distribution model, aiming a higher challenge. We used $\alpha=0.5$ for all methods with boundary polarity constraints.

\section{A. Experiments of COIFT without adjustable width}

The experiment was conducted using a robot user, as proposed by Gulshan et al. [24], to simulate user interaction by placing brush strokes automatically to iteratively perform the segmentation task. We used 40 slice images from CT cervical spine studies of 10 subjects to segment the spinalvertebra (Figures 6g-i). Figure 6 shows segmentation results by OIFT [18] and COIFT for the same user-selected markers, making clear the advantages of COIFT. Figure 7 shows the experimental curve using the robot user, which confirms the same results.

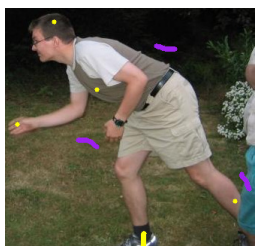

(a)

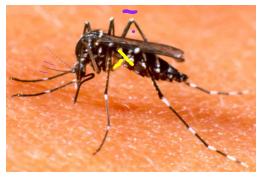

(d)

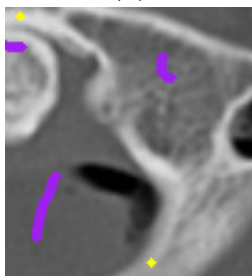

(g)

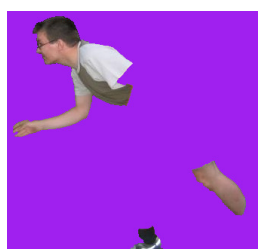

(b)

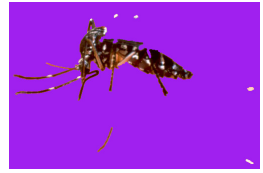

(e)

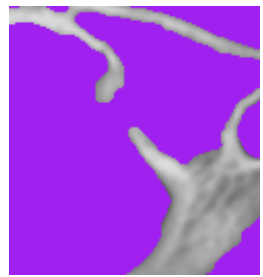

(h)

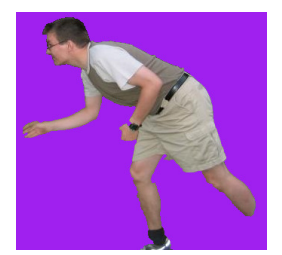

(c)

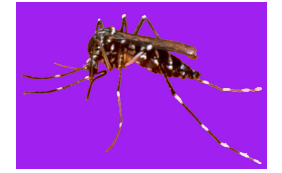

(f)

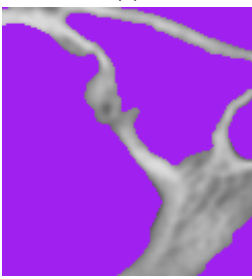

(i)
Fig. 6. (a,d,g) Input images with user-selected markers. (b,e,h) Segmentation without connectivity constraints. (c,f,i) The proposed results by COIFT guarantee connected objects.

\section{B. Experiments of COIFT with adjustable width}

Since segmentation errors of objects with thin and elongated parts do not result in significant differences in the evaluation measure using the Dice Coefficient due to the small number of pixels contained in these regions. In our experiments, we used the Boundary Error ${ }^{1}$ as a comparison measure between the evaluated methods in order to better differentiate their performances. Also, a second approach was considered to automatically get the sets of seeds, that simulates the selection

\footnotetext{
${ }^{1}$ The error was computed as the mean distance of the ground truth boundary from the delineated boundary.
} 




Fig. 7. Spinal-vertebra mean accuracy curve by a robot user.

of seeds with a large brush in the bigger parts of the object and a small brushstroke at the extreme parts of its thin and elongated parts. The skeleton extremes together with the erosion of the object in the ground truth of the images were used to obtain the internal seed set $\mathcal{S}_{1}$ and in the case of the external seed set $\mathcal{S}_{0}$ only the erosion of the background was computed for the same erosion radius considered to obtain $\mathcal{S}_{1}$.

In our experiments with COIFT with adaptable width considering the handling of energy ties, we used two image datasets composed of 100 images of birds and 130 images of spiders, insects and another invertebrates ${ }^{2}$. The ground truth for these images was generated by manual segmentation and it is publicly available to the community 3

Figures 8 and 9 show the mean error curves to segment the dataset of birds and the dataset of spiders, insects and another invertebrates, respectively. Note that ORFC+GC (green curve) [22], [23] did not perform well in these datasets because it was not able to segment some thin and elongated parts of the objects.

\section{CONClusion}

In this work, we have as main results: (1) A novel method called Connected Oriented Image Foresting Transform (COIFT), that support a user-controllable minimum width of the connectivity constraint. We also improved the handling of ties in COIFT energy formulation and discussed its application to avoid objects with holes, by applying the connectivity constraint for the background seeds. The new method, successfully incorporates connectivity constraints on OIFT, preserving its low time complexity $O(N=|\mathcal{V}|$ ) (when $\mathcal{Q}$ is implemented using bucket sorting [9]), since it requires only four executions of the IFT algorithm. (2) The theoretical analysis of a particular case of the cost function of the Riverbed method that guarantees an optimal result according to a graph-cut measure [25]. (3) The theoretical analysis of the

\footnotetext{
${ }^{2}$ These images are released under Creative Commons $\mathrm{CC} 0$ into the public domain, available at the web site https://pixabay.com/

${ }^{3}$ URL: http://www.vision.ime.usp.br/ lucyacm/thesis/coift.html
}

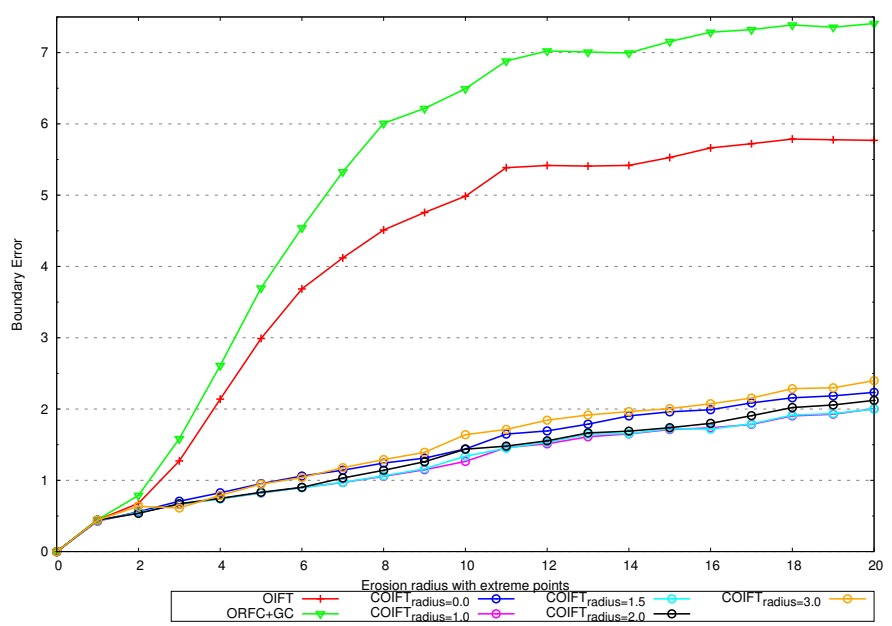

Fig. 8. The mean error curve (boundary error) for the segmentation of the dataset of birds.

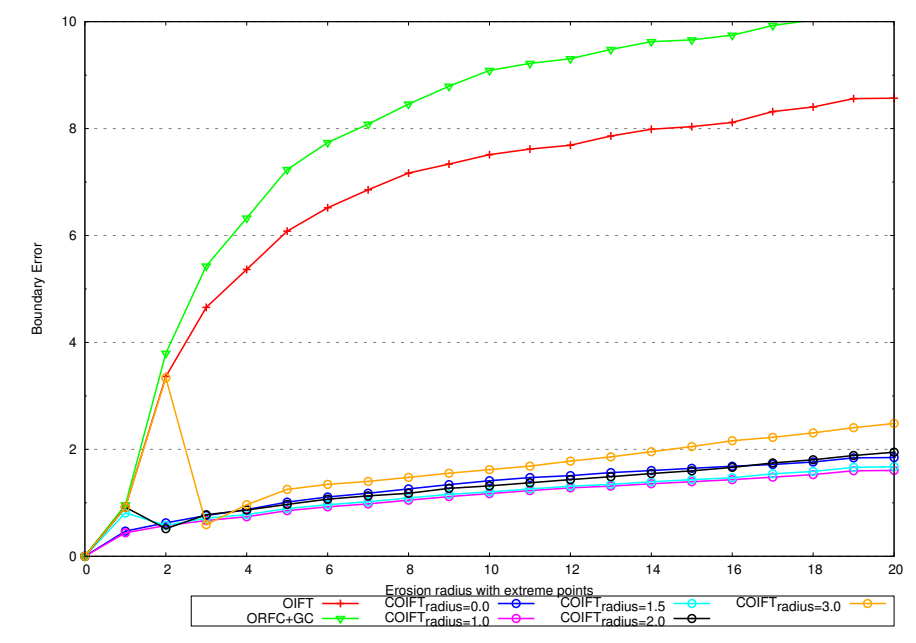

Fig. 9. The mean error curve (boundary error) for the segmentation of the dataset of spiders, insects and another invertebrates.

IFT definition, pointing out a failure in its original PAMI paper and presenting sufficient conditions for its correctness [26]. (4) The design of three new ground truth datasets from 280 public images 4 , which contain objects with thin and elongated parts, available to the community 5 (5) Four conference papers were published in international events of high regard [19], [21], [25], [26]. This work also received the best doctoral thesis award at WVC2018 - XIV Workshop de Visão Computacional.

\section{ACKNOWLEDGMENT}

Thanks to CNPq (308985/2015-0, 305381/2012-1, 486083/2013-6, FINEP 1266/13), FAPESP grant \# 2011/50761-2, CAPES, and NAP eScience - PRP - USP for funding.

\footnotetext{
${ }^{4}$ These images are released under Creative Commons $\mathrm{CC} 0$ into the public domain, available at the web site https://pixabay.com/

${ }^{5} \mathrm{URL}$ : http://www.vision.ime.usp.br/ lucyacm/thesis/coift.html
} 


\section{REFERENCES}

[1] L. Mansilla, "Object segmentation by oriented image foresting transform with connectivity constraints," Ph.D. dissertation, Department of Computer Science, Institute of Mathematics and Statistics University of São Paulo (IME-USP), São Paulo, SP, Brazil, Aug 2018, in Portuguese. [Online]. Available: http://www.teses.usp.br/teses/ disponiveis/45/45134/tde-01102018-120427/en.php

[2] L. Grady, "Random walks for image segmentation," IEEE Trans. Pattern Anaysis and Machine Intelligence, vol. 28, no. 11, pp. 1768-1783, 2006.

[3] Y. Boykov and G. Funka-Lea, "Graph cuts and efficient N-D image segmentation," Intl. Jrnl. of Comp. Vision, vol. 70, no. 2, pp. 109-131, 2006.

[4] X. Bai and G. Sapiro, "Distance cut: Interactive segmentation and matting of images and videos," in Proc. of the IEEE Intl. Conf. on Image Processing, vol. 2, 2007, pp. II - 249-II - 252.

[5] K. Ciesielski, J. Udupa, P. Saha, and Y. Zhuge, "Iterative relative fuzzy connectedness for multiple objects with multiple seeds," Computer Vision and Image Understanding, vol. 107, no. 3, pp. 160-182, 2007.

[6] J. Cousty, G. Bertrand, L. Najman, and M. Couprie, "Watershed cuts: Thinnings, shortest path forests, and topological watersheds," Trans. on Pattern Analysis and Machine Intelligence, vol. 32, pp. 925-939, 2010.

[7] K. Ciesielski, J. Udupa, A. Falcão, and P. Miranda, "A unifying graphcut image segmentation framework: algorithms it encompasses and equivalences among them," in Proc. of SPIE on Medical Imaging: Image Processing, vol. 8314, 2012.

[8] C.Couprie, L.Grady, L.Najman, and H.Talbot, "Power watersheds: A unifying graph-based optimization framework," IEEE Transactions on Pattern Analysis and Machine Intelligence, vol. 99, no. 7, pp. 1384 1399, Jul 2010.

[9] A. Falcão, J. Stolfi, and R. Lotufo, "The image foresting transform: Theory, algorithms, and applications," IEEE Transactions on Pattern Analysis and Machine Intelligence, vol. 26, no. 1, pp. 19-29, 2004.

[10] S. Vicente, V. Kolmogorov, and C. Rother, "Graph cut based image segmentation with connectivity priors," in Computer Vision and Pattern Recognition, 2008. CVPR 2008. IEEE Conf. on, June 2008, pp. 1-8.

[11] Y. Zeng, D. Samaras, W. Chen, and Q. Peng, "Topology cuts: A novel min-cut/max-flow algorithm for topology preserving segmentation in nd images," Computer Vision and Image Understanding, vol. 112, no. 1, pp. 81 - 90, 2008, special Issue on Discrete Optimization in Computer Vision. [Online]. Available: http://www.sciencedirect.com/ science/article/pii/S1077314208001094

[12] S. Nowozin and C. H. Lampert, "Global interactions in random field models: A potential function ensuring connectedness," SIAM Journal on Imaging Sciences, vol. 3, no. 4, pp. 1048-1074, 2010. [Online]. Available: http://dx.doi.org/10.1137/090752614

[13] O. Lézoray and L. Grady, Image Processing and Analysis with Graphs: Theory and Practice. California, USA: CRC Press, 2012.

[14] K. Ciesielski, A. Falcão, and P. Miranda, "Path-value functions for which dijkstra's algorithm returns optimal mapping," Journal of Mathematical Imaging and Vision, Feb 2018. [Online]. Available: https://doi.org/10.1007/s10851-018-0793-1

[15] K. Ciesielski and J. Udupa, "Affinity functions in fuzzy connectedness based image segmentation i: Equivalence of affinities," Computer Vision and Image Understanding, vol. 114, no. 1, pp. 146-154, Jan 2010.

[16] P. Miranda, A. Falcão, and J. Udupa, "Synergistic arc-weight estimation for interactive image segmentation using graphs," Computer Vision and Image Understanding, vol. 114, no. 1, pp. 85-99, Jan 2010.

[17] L. Mansilla and P. Miranda, "Image segmentation by oriented image foresting transform: Handling ties and colored images," in 18th Intl. Conf. on Digital Signal Processing, Greece, Jul 2013, pp. 1-6.

[18] P. Miranda and L. Mansilla, "Oriented image foresting transform segmentation by seed competition," IEEE Transactions on Image Processing, vol. 23, no. 1, pp. 389-398, Jan 2014.

[19] L. Mansilla, P. Miranda, and F. Cappabianco, "Oriented image foresting transform segmentation with connectivity constraints," in Image Processing (ICIP), 2016 23rd IEEE International Conference on, Phoenix, Arizona, USA, Sept 2016, pp. 2554-2558.

[20] P. Miranda, A. Falcao, and T. Spina, "Riverbed: A novel user-steered image segmentation method based on optimum boundary tracking," Image Processing, IEEE Transactions on, vol. 21, no. 6, pp. 3042-3052, June 2012.
[21] L. Mansilla and P. Miranda, "Oriented image foresting transform segmentation: Connectivity constraints with adjustable width," in Graphics, Patterns and Images (SIBGRAPI), 2016 29th SIBGRAPI Conference on, São José Dos Campos, SP, Brazil, Oct 2016, pp. 289-296.

[22] H. H. Bejar and P. A. Miranda, "Oriented relative fuzzy connectedness: Theory, algorithms, and its applications in hybrid image segmentation methods," EURASIP Journal on Image and Video Processing, vol. 2015, no. 21, Jul 2015.

[23] K. C. Ciesielski, P. Miranda, A. Falcão, and J. K. Udupa, "Joint graph cut and relative fuzzy connectedness image segmentation algorithm," Medical Image Analysis (MEDIA), vol. 17, no. 8, pp. 1046-1057, 2013.

[24] V. Gulshan, C. Rother, A. Criminisi, A. Blake, and A. Zisserman, "Geodesic star convexity for interactive image segmentation," in Proc. of Computer Vision and Pattern Recognition, 2010, pp. 3129-3136.

[25] H. Bejar, L. Mansilla, and P. Miranda, "Efficient unsupervised image segmentation by optimum cuts in graphs," in 23rd Iberoamerican Congress on Pattern Recognition (CIARP), vol. LNCS 11401, Madrid, Spain, Nov 2018, pp. 359-367.

[26] M. Condori, L. Mansilla, and P. Miranda, "Bandeirantes: A graph-based approach for curve tracing and boundary tracking," in Mathematical Morphology and Its Applications to Signal and Image Processing, vol. LNCS 10225. Fontainebleau, France: Springer International Publishing, May 2017, pp. 95-106. 